REVIEW ARTICLE

\author{
S.A. Mandelstam
}

\section{Challenges of the Anatomy and Diffusion Tensor Tractography of the Meyer Loop}

\begin{abstract}
SUMMARY: This review addresses the complex and often controversial anatomy of the anterior bundle of the OR, also known as the Meyer loop. Before the advent of MR imaging, 2 main types of studies attempted to ascertain the "safe" distance for anterior temporal lobe resection to avoid postsurgical VFDs. There were those based first on postoperative VFD correlation and second on anatomic dissection studies. In the past decade, noninvasive diffusion MR imaging-based tractography techniques have been developed in an attempt to elucidate white matter connectivity. Although many of these techniques are still experimental, there are some clinical situations for which they may prove to be very helpful if properly performed and validated. The motivation for this review was to improve the outcome of patients with TLE undergoing temporal lobectomy: Would having anatomic information about the OR available to the neurosurgeon decrease the risk of postsurgical VFDs?
\end{abstract}

ABBREVIATIONS: $\mathrm{ATL}=$ anterior temporal lobectomy; $\mathrm{CSD}=$ constrained spherical deconvolution; $\mathrm{DT}-\mathrm{FT}=$ diffusion tensor fiber tractography; DTI = diffusion tensor imaging; $F A=$ fractional anisotropy; $L G B=$ lateral geniculate body; $L G N=$ lateral geniculate nucleus; $O R=$ optic radiations; TLE = temporal lobe epilepsy; TP = temporal pole; VFD = visual field deficit
$T$ emporal lobectomy has become a common and successful form of epilepsy surgery, which can stop the occurrence of TLE in most appropriate patients who are refractory to medical treatment. A common complication of ATL is the occurrence of a postoperative VFD due to disruption of the OR, specifically the anterior bundle, the Meyer loop. Unfortunately, the extent of a postoperative VFD cannot be accurately predicted by conventional MR imaging or from the extent of the resection performed. Risk of damage to the OR is substantial because the tracts are not separately visible under the operating microscope. As the Meyer loop transmits visual information from the contralateral superior field of both eyes, damage will cause homonymous superior quadrantanopia. ${ }^{1}$ The reported incidence of postoperative VFD varies from $68 \%$ to $100 \%$ of patients undergoing ATL. A study in Wales found that only $50 \%$ of 14 patients post-temporal lobectomy would be able to pass the visual examination required to drive. ${ }^{2}$ In Australia, a person with a quadrantanopia or hemianopia is ineligible for an unconditional driver's license according to the "Medical Standards for Licensing and Clinical Management Guidelines" of the Austroads document. ${ }^{3}$ Ironically, a patient may be freed of driving constraints due to epilepsy yet be relegated to a lifetime prohibition of driving due to an acquired VFD.

The scope of this review is to revisit the anatomy of the OR with emphasis on the anterior fiber bundle, the Meyer loop, and its controversial relationship to the TP. The first part of the review will concentrate on the anatomic dissection and

From the Florey Neuroscience and Brain Research Institutes, Melbourne Brain Centre, Heidelberg, Victoria, Australia; and Department of Medical Imaging, Royal Children's Hospital and University of Melbourne, Parkville, Victoria, Australia.

Please address correspondence to Simone A. Mandelstam, MD, Department of Medical Imaging, 2nd Floor, Royal Children's Hospital, Flemington Rd, Parkville 3052, Victoria, Australia; e-mail: simone.mandelstam@rch.org.au

Indicates open access to non-subscribers at www.ajnr.org

http://dx.doi.org/10.3174/ajnr.A2652 postsurgical studies for determining the position of the anterior aspect of the loop. Subsequently, the focus will shift to the utility of DT-FT techniques in the identification of the boundaries of the Meyer loop. Detailed technical MR imaging physics is beyond the scope of this review and will not be addressed.

\section{OR Anatomy}

The OR consist of 4 white matter fiber bundles. The anterior bundle or the Meyer loop (Meyer 1907) ${ }^{4}$ projects from the LGB and runs anteriorly across the superior aspect of the anterior tip of the ipsilateral temporal horn before making a sharp turn to pass posteriorly along the wall of the lateral ventricle to converge on the lower lip of the calcarine fissure. ${ }^{5,6}$ The central bundle leaves the LGB in a lateral direction and follows posteriorly along the lateral ventricular wall to the visual cortex. The dorsal bundle extends directly posterior to meet the upper part of the calcarine cortex (Fig 1).

\section{Surgical Studies}

The boundary of the anterior fibers of the Meyer loop and its relationship to the TP has been controversial. Older studies used intraoperative estimates of resection size or brain dissection (Table). There was no consistency among the reported locations, which varied from 30 to $45 \mathrm{~mm}$ posterior to the TP. $^{7-9}$ In 1954, Penfield ${ }^{10}$ stated that resections extending $<6 \mathrm{~cm}$ posterior to the tip of the TP were "not likely" to result in VFD. These studies relied on the surgeon to subjectively estimate the resection size and the anterior extent of the Meyer loop in the absence of neuroimaging. The resection sizes were probably overestimated with underestimation of the Meyer loop because this would not be visualized surgically. This inaccuracy could explain why more recent studies by using different methodologies show smaller measurements. In addition, VFDs have not been assessed in a consistent manner. Older studies relied on the patient verbally reporting the deficit, which is very unreliable. The incidence of VFDs seems to be increasing because of the increased sensitivity of automated perimetry for small defects compared with kinetic perimetry. ${ }^{2}$ 

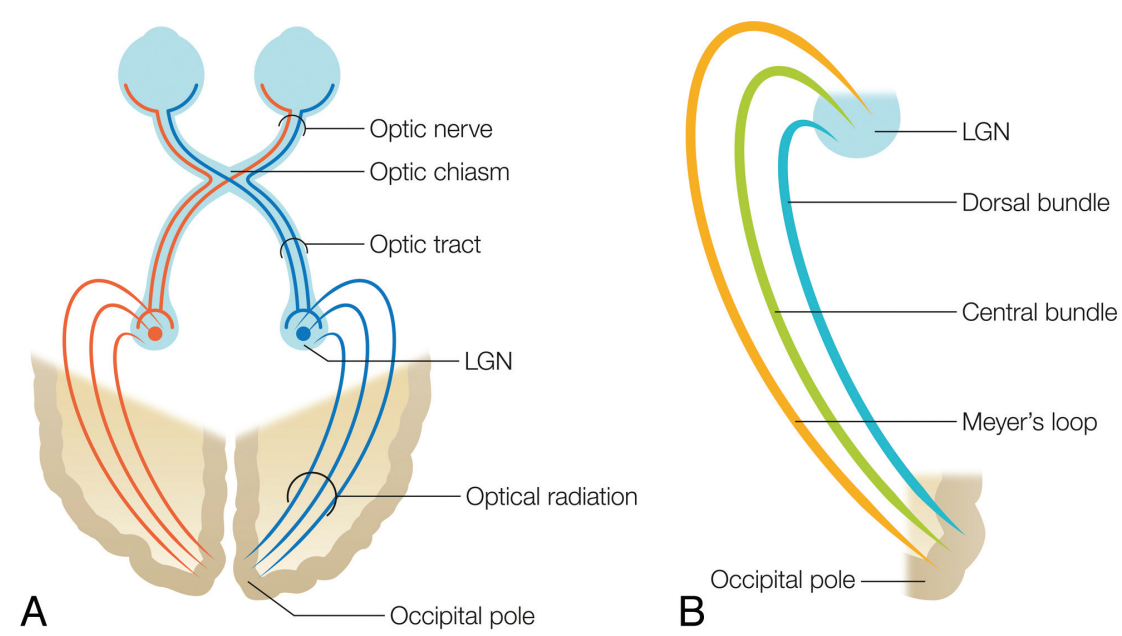

Fig 1. Schematic drawing of the human visual system. $A$, Right- and left-hemispheric fiber pathways. $B$, OR comprising the anterior bundle, the Meyer loop (orange), the central bundle (green), and the dorsal bundle (turquoise).

\begin{tabular}{|c|c|c|}
\hline \multicolumn{3}{|c|}{ Distance between the anterior boundary of Meyer loop and the temporal pole tip as reported using differing techniques, 1954-2010 } \\
\hline & Distance from Anterior Edge of the Meyer & \\
\hline Author/Year & Loop to Temporal Pole Tip (mm) & Measurement Technique \\
\hline Penfield $1954^{10}$ & 60 & Surgical resection, VFD \\
\hline Bjork and Kugelberg $1957^{7}$ & 30 & Surgical resection, VFD, 96\% partial Goldmann perimetry \\
\hline Falconer and Wilson $1958^{8}$ & 45 & $\begin{array}{l}\text { Surgical resection, VFD, } 64 \% \text { complete and 36\% } \\
\text { incomplete Bjerrum }\end{array}$ \\
\hline Marino and Rasmussen $1968^{9}$ & 45 & $\begin{array}{l}\text { Surgical resection, VFD, } 14 \% \text { complete and } 52 \% \\
\text { incomplete Aimark and Tangent }\end{array}$ \\
\hline Ebeling and Reulen $1988^{11 *}$ & $27(22-37)$ & Cadaver dissection (Klingler technique), 50 hemispheres \\
\hline Krolak-Salmon et al $2000^{21}$ & 20-31.3 & $\begin{array}{l}\text { MR imaging and automated static perimetry, } 28 \% \text { mild } \\
28 \% \text { moderate }\end{array}$ \\
\hline Kier et al $2004^{12}$ & Anterior fibers do not reach temporal horn tip & "Anatomic dissection tractography" \\
\hline Rubino et al $2005^{6}$ & $25(22-30)$ & Cadaver dissection (Klingler technique), 40 hemispheres \\
\hline Barton et al $2005^{25}$ & $24(18-36)$ & $\begin{array}{l}\text { MR imaging and automated Goldmann perimetry, 100\% } \\
\text { had VFD }\end{array}$ \\
\hline Yamamoto et al $2005^{18}$ & $37.3 \pm 2.5$ & DT-FT \\
\hline Choi et al $2006^{5}$ & $31.4(28-34)$ & Cadaver dissection (Klingler technique), 10 hemispheres \\
\hline Nilsson et al $2007^{14}$ & $44(34-51)$ & DT-FT \\
\hline Taoka et al $2008^{26}$ & $36.6(30-43.2)$ & DT-FT \\
\hline Chen et al $2009^{27}$ & $32.1(20.9-51.5)$ & DT-FT \\
\hline Yogarajah et al $2009^{32}$ & Patients 24-43, controls 24-47 & DT-FT \\
\hline Wang et al $2010^{28}$ & 36 (mean) & DT-FT \\
\hline
\end{tabular}

\footnotetext{
* Criterion standard study.
}

\section{Anatomic Dissection Studies}

The more recent group of anatomic studies uses the Klingler fiber dissection technique in cadaveric brains of previously healthy patients to look at the anatomy of the Meyer loop. This technique involves fixing a cadaveric brain in formalin, freezing it, and thawing it before dissection. The freezing and thawing of the cadaveric brain allow ice crystals to form between myelinated nerve fibers, thereby separating them and facilitating dissection (Fig 2).5,6,11 Ebeling and Reulen (1988) ${ }^{11}$ dissected 25 brains and reported that the distance between the tip of the temporal lobe and the anterior edge of the Meyer loop is $27 \pm 3.5 \mathrm{~mm}$ with a range of $22-37 \mathrm{~mm}$. They stated that the average location of the anterior edge of the Meyer loop is $5 \pm$ $3.9 \mathrm{~mm}$ anterior to the temporal horn tip. These studies also demonstrated differing distances from the OR to the TP (Fig 3). Choi et $\mathrm{al}^{5}$ found an average of $31.4 \mathrm{~mm}$, and Rubino et $\mathrm{al}^{6}$ found an average of $25 \mathrm{~mm}$, which is similar to the measurements of Ebeling and Reulen. Kier et $\mathrm{al}^{12}$ combined dissection and MR imaging, a method they termed "anatomic dissection tractography," and described the anterior extent of the Meyer loop to be at the level of the amygdala and not reaching the level of the temporal horn tip. However, Sincoff et $\mathrm{al}^{13}{ }^{13}$ in a dissection study, reported that the anterior bundle of the OR did cover the anterior tip of the lateral aspect of the temporal horn.

What becomes apparent looking at the different and conflicting results of these studies is that there is great variability, which makes it difficult to give a generic recommendation to the neurosurgeon on the "safe" length of anterior temporal lobe that can be resected without causing a VFD. Potential sources of bias include relatively small sample sizes, interobserver and intersurgical variability, and different methodologies. Dissection can only be used ex vivo and does not reliably 


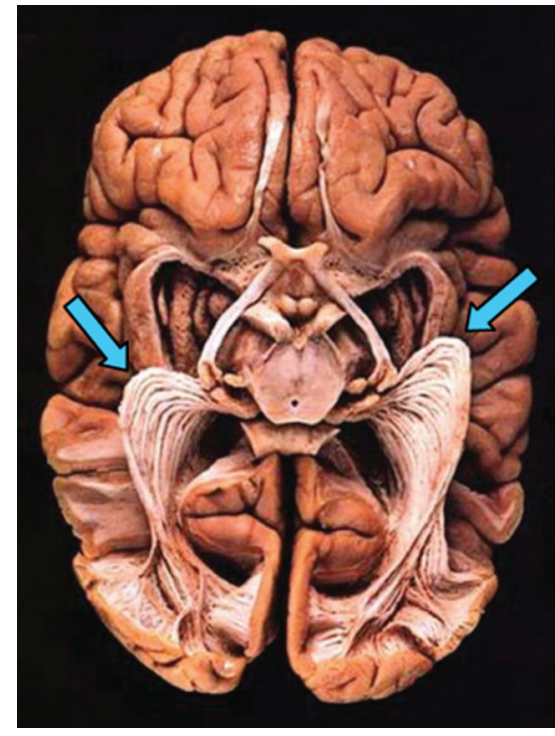

Fig 2. The $O R$ exposed (virtual hospital). A brain viewed from below is prepared by using the Klingler fiber dissection technique. The Meyer loop, the anterior extension into the temporal lobe, is indicated by the arrows. Reproduced with permission from the Journal of Vision and Sherbondy et al. ${ }^{31}$

separate 1 fiber system from another. In fact, dissection of 1 white matter tract may lead to destruction of other tracts. Formalin shrinks brain tissue, which could influence measurements of anatomic distances, while the water freezing technique has been found to cause fissuring of the specimen. ${ }^{14}$ Despite all these differences, there is likely an underlying fundamental issue, which is the intersubject variability of the OR. There may even be significant hemispheric asymmetries within each individual, postulated to be due to expanded language areas in the left posterior temporal lobe displacing the OR on that side. ${ }^{15,16}$

\section{Modern Imaging}

The next part of this review will focus on the anatomy of the Meyer loop as demonstrated by modern imaging techniques. Unfortunately conventional MR imaging sequences cannot differentiate different white matter tracts. However, an MR imaging-based method for measuring the course of white matter tracts has been developed. This is known as DTI. Among the analyzing methods for DTI, DT-FT has been reported as robust for visualizing and evaluating white matter fiber direction and connectivity in the brain. ${ }^{17-19}$

\section{Overview of DTI}

DTI is an MR imaging technique that can be used to characterize the directional properties of the diffusion of water molecules. It is based on the principle that diffusion is directed by anatomic microstructures (ie, white matter fibers). ${ }^{17-19}$

DT-FT offers the only noninvasive method for measuring the course of white matter tracts in vivo; however, DT-FT estimates have ongoing difficulty identifying the OR, especially the Meyer loop. ${ }^{18}$

DTI indirectly evaluates the integrity of white matter by measuring water diffusion and directionality in 3D. From information about direction of diffusion with a minimum of 6 different nonparallel directions, one can calculate the diffusion tensor and derive FA from this. FA values range from zero (maximal isotropic diffusion) to 1 (maximal anisotropic diffusion). Major, medium, and minor eigenvalues specify rates of diffusivity along each of 3 orthogonal axes of a diffusion ellipsoid. ${ }^{17}$ The direction of the largest eigenvector is the direction of greatest diffusivity and is assumed to align with the direction of fiber bundles. This is important in regions with densely packed axons such as white matter. Diffusion characteristics of a tissue provide information on its structural properties.

Data may be analyzed with a region-of-interest or voxelbased approach. For the voxel-based approach, all brains need to be normalized in common space. Although this is a statistically rigorous method, there may geometric distortions, eddy currents, magnetic susceptibility, patient motion, or image noise resulting in reduced sensitivity.

Region-of-interest approaches are also limited by user variability because regions are manually outlined. To decrease user variability, the region of interest can be used as a seed point for tractography with a threshold for FA applied and the resulting voxels can be used for calculation.

\section{$D T-F T$}

DT-FT is the only available noninvasive technique that can delineate white matter tracts in vivo. Directional anisotropy information in each voxel provided by DTI is used to generate virtual maps of white matter tracts. Different algorithms determine how voxels should be connected according to their anisotropy and direction. ${ }^{17}$

There has been some confusion about the best technique, with 2 approaches described in the literature.

1. Deterministic: fiber assignment by continuous tracking. From a seed region of interest, tracking follows the largest tensor in each voxel and connects the voxels according to specific thresholds for minimum FA and maximum change of direction between 2 voxels.

2. Probabilistic: fast-marching tractography and probabilistic index of connectivity. These methods find the energetically most favorable pathway between 2 voxels. The probability of connectivity between voxels from a seed point is calculated. ${ }^{20}$

The criterion standard for preoperative detection of the course of the OR is based on the gross dissection technique on frozen formalin-fixed tissue. The study by Ebeling and Reulen ${ }^{11}$ has been used as the criterion standard for OR location measurements in 2 DT-FT methods, ${ }^{14,18}$ and the data have been confirmed by additional dissection ${ }^{5,6}$ and clinical studies. $^{21}$ Krolak-Salmon et $\mathrm{al}^{21}$ reported that 15 of 18 patients presented with a postoperative visual field loss. They reported 2 cases in which the resection was limited to $20 \mathrm{~mm}$ from the TP but still produced a partial quadrantanopia.

The earlier reports of DT-FT of the OR were performed on very few patients. ${ }^{14,18,22,23}$ Yamamoto et al $^{18}$ did basic deterministic fiber tracking on 5 volunteers and found difficulty identifying the OR and particularly the anterior extent of the Meyer loop. Powell et $\mathrm{al}^{22}$ had similar issues performing fiber tracking on 2 patients, of whom 1 had an intact Meyer loop and no postoperative VFD and the other had a disrupted Meyer loop with a postoperative VFD. Powell et al used 54 gradients with the assumption that this would improve the fiber tracking. Yamamoto et al in $2007^{19}$ experimented with 


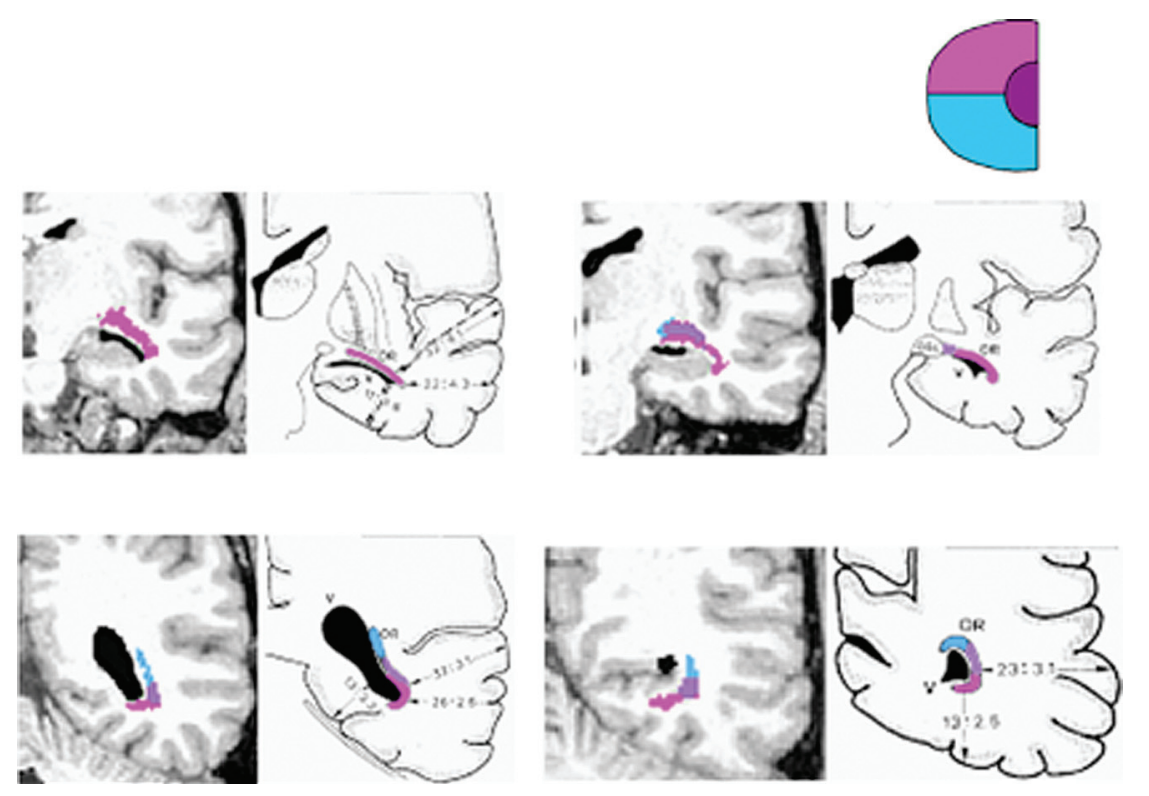

Fig 3. Coronal sections showing the position of the direct, central, and the Meyer loop bundles within the OR. Each panel compares an MR image and estimated fibers (left) with illustrations from a dissection study (Ebeling and Reulen, 1988 ${ }^{10}$ ) (right). The positions of the OR bundles are shown by the color overlays, and the expected visual information carried by these bundles is indicated by the inset (upper right). Reproduced with permission Journal of Vision and Sherbondy et al. ${ }^{31}$

using $6,12,40$, and 81 gradients and found that there was no significant effect on visualization of the OR. They concluded that 6 directions are thus sufficient. In fact, this is true for the deterministic approach. No matter how many directions are used, the major eigenvector will be the same, and there is no true indication of the confidence that can be assigned to a particular tracking result. Therefore, the pathway may have no or little correlation with the underlying anatomy. The adoption of visualization strategies gives the impression that the viewer is looking at something "real," and this could lead to serious errors. ${ }^{24}$ This is far more likely to be the case in a region where the fiber tracts bend and loop such as in the Meyer loop because these methods are inherently unable to take into account crossing fibers.

The extent of brain shift related to surgery could also cause errors in the attempts to estimate the status of fiber tracts according to the resection extent alone..$^{22,25}$ Despite some success, DT-FT estimates cause problems identifying the OR and particularly the Meyer loop section. ${ }^{18,22}$

Both the Nilsson and Yamamoto groups estimated the mean anterior position of the Meyer loop to be at least $1 \mathrm{~cm}$ posterior to estimates from dissection studies. ${ }^{14,18}$ For example, Nilsson et al ${ }^{14}$ described the temporal horn as being $1.5 \mathrm{~cm}$ anterior to the Meyer loop. They suggested that the discrepancy may be due to misidentification of the fibers during dissection or errors in the dissection estimates of absolute distances. Distance errors are not an adequate explanation because the anatomic descriptions are detailed, with 1 study stating that the "anterior tip of the temporal horn was covered by the anterior optic radiation along its lateral half"13 and another confirming that in all of their specimens, "the anterior edge of the Meyer loop reached the tip of the temporal horn." 6

Taoka et $\mathrm{al}^{26}$ looked retrospectively at the data of 14 patients with hippocampal sclerosis who underwent temporal lobectomy. They were divided into 4 groups according to the size of the acquired VFD with group A having no deficit. Trac- tography of the uncinate fasciculus was used to help recognize the most anterior point of the Meyer loop with the assumption that the loop is immediately behind the uncinate fasciculus. Although they claimed to have obtained tractography of the Meyer loop in "all the cases," they also stated that there was no differentiating the loop from the uncinate fasciculus in 50\% of cases. $^{26}$ Therefore, one would have to question the accuracy of their Meyer loop reconstructions. Although they reportedly tried to evaluate postsurgical tractography of the OR, in most cases tractography could not be drawn. This problem was theorized to be due to edema or gliosis within adjacent tissue, which would change the FA and thus lead to termination of the tracking. However, Chen et $\mathrm{al}^{27}$ made the valid point that there is a shift of the fiber tracts intraoperatively in vertical and horizontal planes at the resection site and that this would have to be factored into postoperative calculations.

Despite the limits of streamlined deterministic tracking, it is fairly simple computationally and seems easy to interpret if the viewer is unaware of the pitfalls. There are groups that continue to use this methodology. A study was recently published on a cohort from Southern China attempting to establish an average local population value for the distance between the anterior tip of the Meyer loop and the TP. Interestingly, the scans were obtained on 16 patients with a variety of neurologic conditions ranging from brain tumors (not in the temporal lobe) to Parkinson disease. The 2 operators doing the analysis were a senior neurosurgeon and a junior radiologist using different software programs. The conclusion was that the distance from the Meyer loop to the TP is $36 \mathrm{~mm}$ in this population, and recommendations were given for surgical planning. ${ }^{28}$ This advice was based on multiple levels of accumulated errors (neurologically abnormal brains, operator and software differences, unvalidated technique, and so forth), and utmost caution would be needed before accepting this as fact.

Hofer et $\mathrm{al}^{29}$ used a new diffusion-weighted sequence with a simple DT-FT algorithm, which is claimed to decrease sus- 

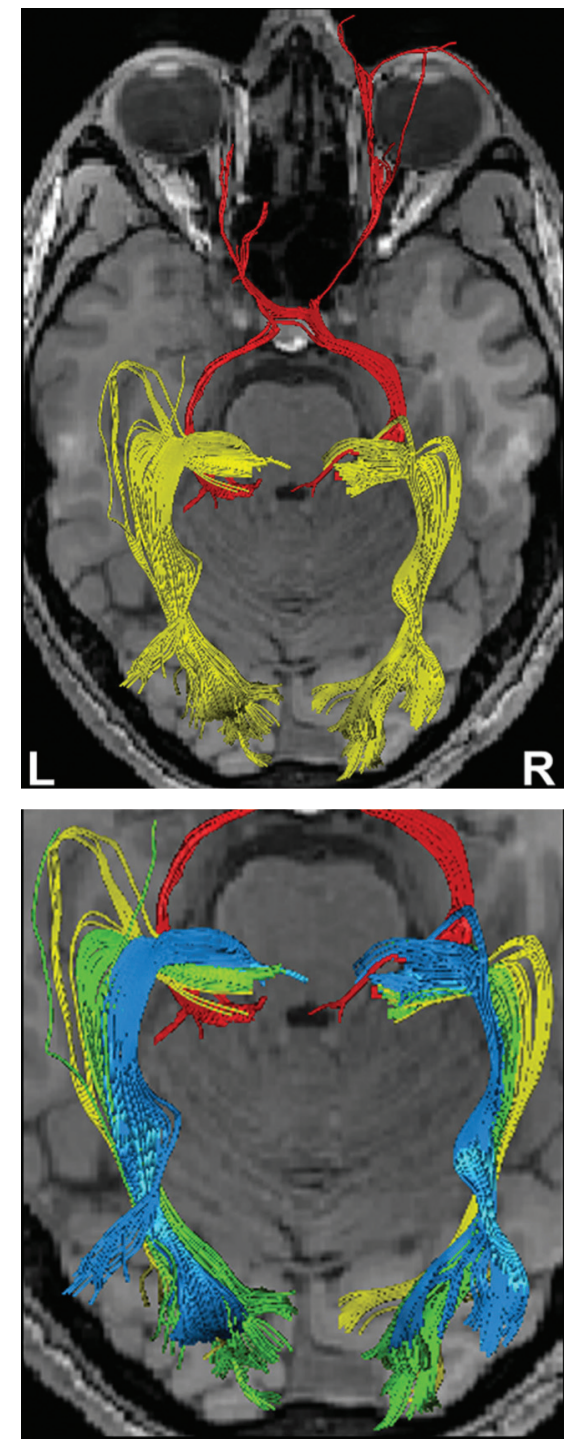

Fig 4. Top: Reconstructions of the optic nerves, tracts (red), and OR (yellow). Bottom: Dissection of the OR into the Meyer loop (yellow), the central bundle (green), and the dorsal bundle (blue). The use of 1.8-mm isotropic voxels reduces the voxel size of a more conventional 2.5-mm acquisition by a factor of 2.7. Nevertheless, this resolution is still not good enough to avoid "kissing" effects between the optic nerve and neighboring eye muscles. Reproduced with permission from Frontiers in Neuroanatomy and Hofer et al. ${ }^{29}$

ceptibility artifacts and geometric distortions and improve spatial resolution. However, their resolution of $1.8 \mathrm{~mm}$ is still not adequate to avoid some recognized artifacts, including false-positive tracts. They then used a region of interest DT-FT method to generate tracking maps of the visual pathway. They reported that "most" fiber bundles could be fully or partially reconstructed in 5/6 subjects. In 1 subject, they were unable to reconstruct any bundles of the OR, and this was ascribed to the close vicinity and predominance of the inferior longitudinal fasciculus and the fronto-occipital fasciculus. ${ }^{29}$ This result is similar to the inability of Taoka et $\mathrm{al}^{26}$ to separate the OR from the uncinate fasciculus as discussed above. These studies continue to give an approximation of the pathways, but none can match the criterion standard of the dissection studies (Figs 4 and 5). Yet, the derived anatomy is thought to be increasingly valid with Kamada et $\mathrm{al}^{23}$ showing, in 2 patients, that real-time perioperative visual-evoked potentials confirmed the accuracy of tractography.

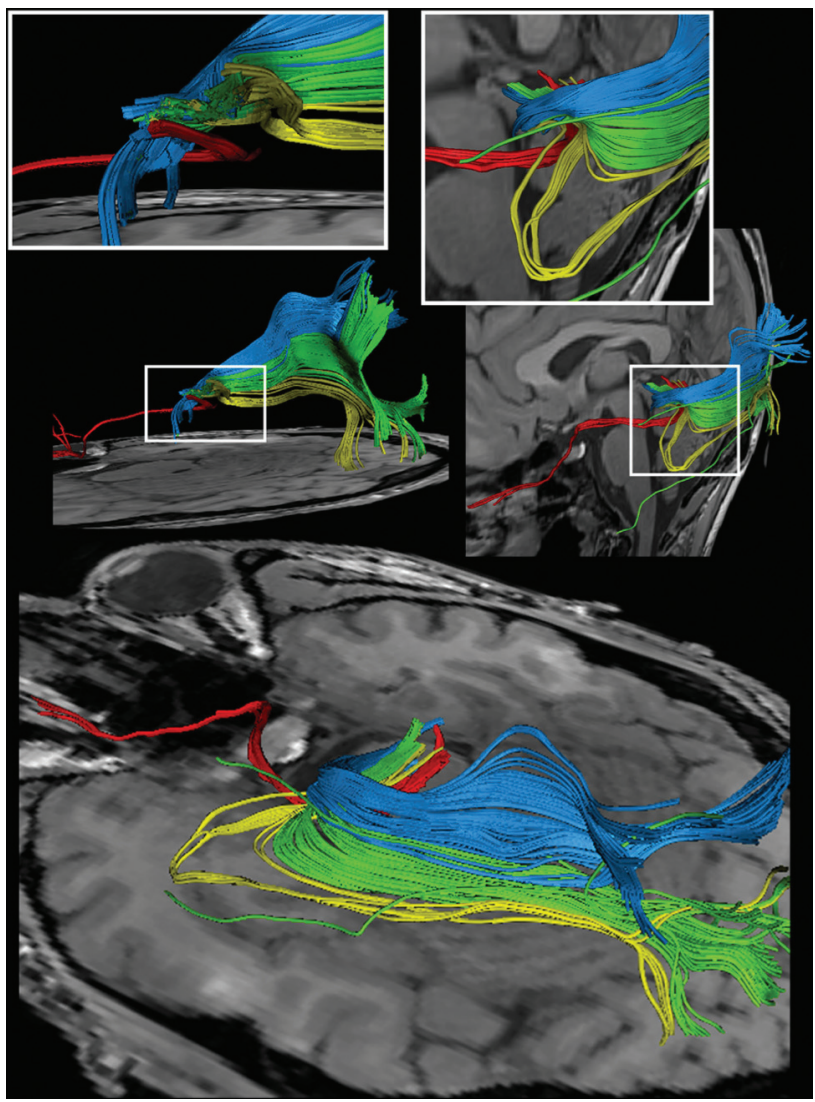

Fig 5. Reconstructions of the optic nerve and tract (red), the Meyer loop (yellow), the central bundle (green), and the dorsal bundle (blue) in different planes. White boxes show magnifications of the area around the LGN. Upper left: Fiber bundles of the right hemisphere. Upper right and bottom: Fiber bundles of the left hemisphere. Reproduced with permission from Frontiers in Neuroanatomy and Hofer et al. ${ }^{29}$

As the high false-negative rate of the streamlined deterministic tracking approach with diffusion tensor data became recognized, new algorithms by using probabilistic methods have been developed to attempt to locate valid pathways. Probabilistic tracking algorithms generate multiple pathways from a given point in space. The end result is a set of multiple pathways (streamlines) passing through the seed point. Counting how many times each voxel in the volume of interest is intersected by a streamline and expressed as a percentage then summarizes this information. The algorithm is run repeatedly to build up a pattern of possible paths through the data. Highangular-resolution diffusion imaging, with large numbers of gradient directions, increases this ability to estimate the probability distributions of fiber directions and has been shown to increase the volume of tracts generated by tractography compared with methods using fewer gradient directions (Fig 6). ${ }^{30}$

Probabilistic tracking results give an indication of the precision of the tracking result but say nothing about accuracy. However, inexperienced observers may still misinterpret these maps. There may appear to be a continuous pattern that is completely inaccurate with regard to true anatomy. ${ }^{24}$ The accuracy and precision for curved paths is lower than that for straight paths due to accumulated error, which is a major problem with tracking a structure that has the configuration of the Meyer loop. Sherbondy et $\mathrm{al}^{31}$ used a probabilistic tracking method on 8 volunteers to identify the "most likely" path be- 


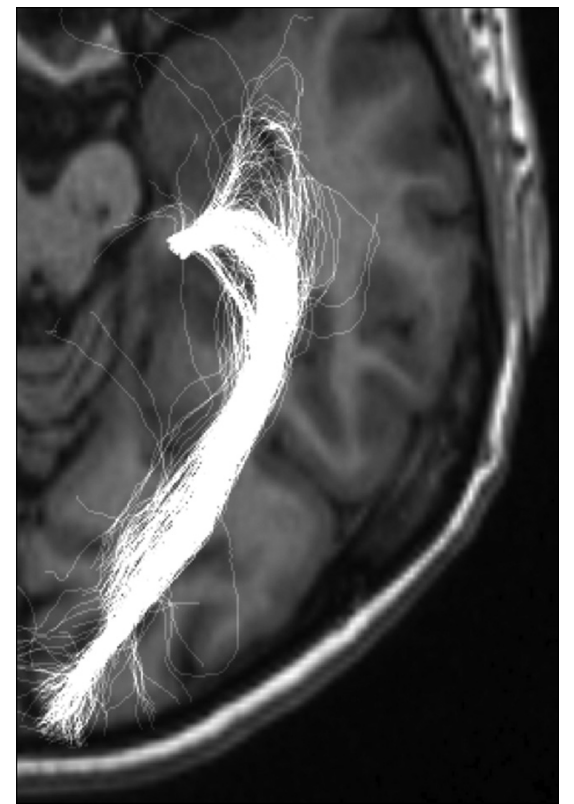

Fig 6. The Meyer loop by using CSD. Courtesy of Alan Connelly, Brain Research Institute, Melbourne, Australia.

tween the lateral geniculate nucleus and the calcarine sulcus. Validity scores were independently assigned to every pathway connecting the LGN region of interest and the calcarine sulcus region of interest. ${ }^{31}$ The findings were nearer to the criterion standard dissection results of Ebeling and Reulen, ${ }^{11}$ with the average distance of the most anterior position of Meyer loop measuring $28 \mathrm{~mm}$ from the TP.

Probabilistic multifiber tractography applied to diffusion MR imaging data acquired at 3T may be better able to cope with crossing and kissing fibers than deterministic models because it allows many more possible local pathway orientations for each DTI sample point. This means that it is less likely to underestimate the anterior extent of the Meyer loop. Yogarajah et $\mathrm{al}^{32}$ combined retrospective probabilistic fiber tracking in 20 controls and 21 postsurgical patients with postoperative assessment of VFDs. Nine of 21 patients had VFDs of up to $87 \%$ of the contralateral superior quadrant. The distances from the tip of the Meyer loop to the TP can be seen in the Table. Resection size was ascertained by using the Barton method, and Goldmann perimetry was performed by a consultant ophthalmologist blinded to the results of tractography and estimates of resection size. Using linear regression analysis, they demonstrated that the distance from the tip of the Meyer loop to the TP and the size of resection were significant predictors of the postoperative VFDs. There was a significant association between the anterior extent of the Meyer loop and whether a patient had a severe $(>50 \%)$ quadrantic VFD $(P=$ $.01)$. If the anterior extent of the loop was $<35 \mathrm{~mm}$ from the $\mathrm{TP}$, patients were more likely to have severe VFDs irrespective of the size of the resection. ${ }^{32}$ This finding is in keeping with the observations of Chen et $\mathrm{al}^{27}$ that the change in the size of the Meyer loop postresection for each subject allowed calculation of the individual preserve ratio for the prediction of the postoperative VFDs. Despite the limitations of the study, which included lack of blinding of the investigators involved in image processing and processing in the coronal plane only, these results are similar to those of the dissection studies and the VFD-based study of Barton et al. ${ }^{25}$ Operator-dependent factors such as variability in seed voxel placement and the choice of connectivity threshold to select between tract and nontract voxels are potential causes of significant variability. This leads to the tracts being "virtual" representations of underlying white matter.

One of the biggest problems with probabilistic tractography methods is lack of validation against a histologic standard, causing difficulty in evaluating individual methods and quantitatively comparing methods. ${ }^{24,30}$ Clatworthy et $\mathrm{al}^{30}$ have started to address this by describing a method of OR tractography using an existing well-validated probabilistic algorithm with the ability to accommodate multiple fiber directions within each voxel. A method of quantitatively assessing the accuracy of tract images is described on the basis of receiver operating characteristic analysis. This compares generated tracts with probabilistic histologic data of the OR in MR imaging space and provides an estimate of the frequencies of both false-positive and false-negative voxels. Seed voxel placement is automated, removing differences between operators as a source of tract image variability. Threshold selection is objective, based on a quantitative comparison of tract images and histologic reference data in stereotaxic space. Their results are described as showing a good match between the resulting binary tract images and the reference data by using a second group to validate the parameters generated in the first group of subjects. Although once again the sample size is small, this study is a good first step toward addressing the issue of validation.

In general, there are limitations common to all existing tractography methods. False-positive tracts can be generated that are not part of the tract of interest. This is relevant when the anterior projections from the OR may actually reflect the inferior occipitofrontal fibers. ${ }^{20}$ Peripheral voxels to the central tract may be represented as having lower probability and may be removed at the thresholding process with inclusion of regions around the central tract, which makes the central tract appear more bulky than it actually is. Voxel values, which represent the probability of tracing a pathway through the diffusion, do not relate in a straightforward way to the anatomic strength of the underlying pathways. ${ }^{24}$ Voxel size used in DTI can be between 1 and $15 \mathrm{~mm}^{3}$, but an axon measures approximately $0.01 \mathrm{~mm}$. Therefore, the signal from 1 voxel represents thousands of axons that may have different directions. Algorithms can have difficulty tracking fibers crossing in a voxel. ${ }^{16}$ Although the probabilities will reflect anatomically meaningful features such as axonal packing density and myelination, they will also be influenced by factors such as the length of the pathway (because probabilities will decay with distance) and tract geometry. ${ }^{30}$

\section{The Future}

At this point in time, to my knowledge, there is no single validated recommended method of DT-FT in the literature. Early attempts at tracking the OR have demonstrated mixed success, though it seems that newer probabilistic methods may be more representative of the OR than deterministic methods. Further work is needed to improve and validate fiber tracking algorithms and to develop multimodal techniques for assessment of brain connectivity. ${ }^{24}$ The anatomic measurements of 


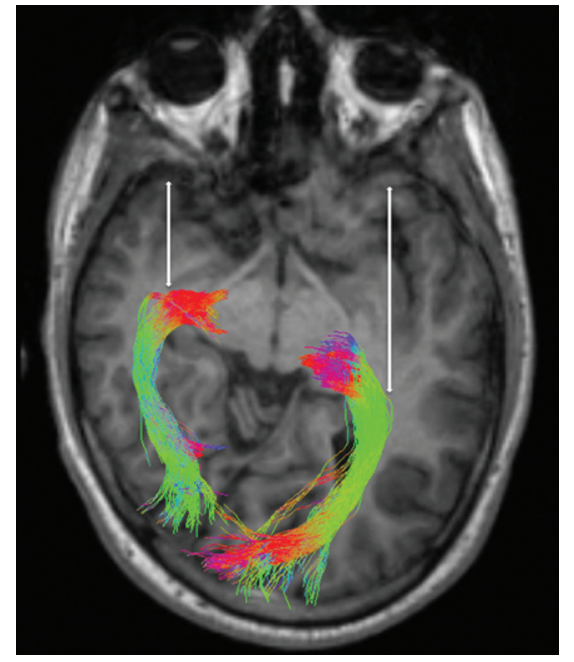

Fig 7. Reconstructed $O R$ by using the probabilistic method of $\mathrm{CSD}^{32}$ in a 17-year-old male adolescent with a small right cerebral hemisphere. Arrows indicate the distances from the Meyer loop to the anterior TPs. Note the differences in position between the right and left OR.

the anterior border of the Meyer loop have been controversial for half a century, and fiber tracking, with its inherent limitations, is not simplifying this issue. The general consensus seems to be that there is great individual variation in this anatomically complex region.

It is always important to bring the patient back into focus. Tractography studies are showing great promise in the delineation of the Meyer loop, and effort should be made to use this information to direct the neurosurgical approach to the individual patient to avoid a VFD or to appropriately counsel the patient regarding a VFD if deemed surgically unavoidable (Fig 7). New tractography algorithms and methods are being devised to deal with the issues of crossing fibers to improve the reliability of the resulting estimates of the fiber orientation density within each voxel. ${ }^{33}$

Prospective clinical studies by using these methods are needed in which the preoperative tractography results are used by the neurosurgeons in modifying the surgical approach. The major questions are the following:

- How accurate are DT-FT-based studies of the OR?

- Can a criterion standard validated tractography algorithm be developed for standardized assessment of the OR?

- Will tractography of the OR significantly decrease the incidence and severity of postoperative VFDs in patients with TLE who undergo ATL?

\section{Acknowledgments}

I acknowledge Shawna Farquharson for the processing of Figure 7.

\section{References}

1. Jacobson DM. The localizing value of a quadrantanopia. Arch Neurol 1997;54:401-04

2. Pathak-Ray V, Ray A, Walters R, et al. Detection of visual field defects in patients after anterior temporal lobectomy for mesial temporal sclerosis: establishing eligibility to drive. Eye 2002;16:744-48

3. Medical standards for licensing and clinical management guidelines: section 23 vision and eye disorders-assessing fitness to drive. Austroads Inc 2006; 23.2.3 and 23.3.1:96-100

4. Meyer A. The connections of the occipital lobes and the present status of the cerebral visual affections. Trans Assoc Am Physicians 1907;22:7-15
5. Choi C, Rubino PA, Fernandez-Miranda JC, et al. Meyer's loop and the optic radiations in the transsylvian approach to the mediobasal temporal lobe. Neurosurgery 2006;59(4 suppl):228-36

6. Rubino PA, Rhoton AL Jr, Tong X, et al. Three-dimensional relationships of the optic radiation. Neurosurgery 2005;57(4 suppl):219-27

7. Bjork A, Kugelberg E. Visual field defects after temporal lobectomy. Acta Ophthalmol (Copenh) 1957;35:210-16

8. Falconer MA, Wilson JL. Visual field changes following anterior temporal lobectomy: their significance in relation to Meyer's loop of the optic radiation. Brain 1958;81:1-14

9. Marino R Jr, Rasmussen T. Visual field changes after temporal lobectomy in man. Neurology 1968;18:825-35

10. Penfield W. Temporal lobe epilepsy. Br J Surg 1954;41:337-43

11. Ebeling U, Reulen H-J. Neurosurgical topography of the optic radiation in the temporal lobe. Acta Neurochir 1988;92:29-36

12. Kier EL, Staib LH, Davis LM, et al. MR imaging of the temporal stem: anatomic dissection tractography of the uncinate fasciculus, inferior occipitofrontal fasciculus, and Meyer's loop of the optic radiation. AJNR Am J Neuroradiol 2004;25:677-91

13. Sincoff EH, Tan Y, Abdulrauf SI. White matter fiber dissection of the optic radiations of the temporal lobe and implications for surgical approaches to the temporal horn. J Neurosurg 2004;101:739-46

14. Nilsson D, Starck G, Ljungberg M, et al. Intersubject variability in the anterior extent of the optic radiation assessed by tractography. Epilepsy Res 2007;77:11-16

15. Wang F, Sun T, Li XL, et al. Diffusion tensor tractography of the temporal stem on the inferior limiting sulcus. J Neurosurg 2008;108:775-81

16. Jeelani $\mathrm{Nu} \mathrm{O}$, Jindahra $\mathrm{P}$, Tamber MS, et al. 'Hemispherical asymmetry in Meyer's Loop: a prospective study of visual-field deficits in 105 cases undergoing anterior temporal lobe resection for epilepsy. J Neurol Neurosurg Psychiatry 2010;81:985-91. Epub 2010 Jun 27

17. Mori S, van Zijl PC. Fiber tracking: principles and strategies-a technical review. NMR Biomed 2002;15:468-80

18. Yamamoto T, Yamada K, Nishimura T, et al. Tractography to depict three layers of visual field trajectories to the calcarine gyri. Am J Ophthalmol 2005;140:781-85

19. Yamamoto A, Miki Y, Urayama S, et al. Diffusion tensor fiber tractography of the optic radiation: analysis with 6-, 12-, 40-, and 81-directional motion-probing gradients-a preliminary study. AJNR Am J Neuroradiol 2007;28:92-96

20. Ciccarelli O, Toosy AT, Parker GJ, et al. Diffusion tractography based group mapping of major white-matter pathways in the human brain. Neuroimage 2003;19:1545-55

21. Krolak-Salmon P, Guenot M, Tiliket C, et al. Anatomy of optic nerve radiations as assessed by static perimetry and MRI after tailored temporal lobectomy. Br J Ophthalmol 2000;84:884-89

22. Powell HW, Parker GJ, Alexander DC, et al. MR tractography predicts visual field defects following temporal lobe resection. Neurology 2005;65:596-99

23. Kamada K, Todo T, Morita A, et al. Functional monitoring for visual pathway using real-time visual evoked potentials and optic-radiation tractography. Neurosurgery 2005;57(1 suppl):121-27

24. Jones DK. Challenges and limitations of quantifying brain connectivity in vivo with diffusion MRI. Imaging in Medicine 2010;2:341-55

25. Barton JJ, Hefter R, Chang B, et al. The field defects of anterior temporal lobectomy: a quantitative reassessment of Meyer's loop. Brain 2005; 128:2123-33

26. Taoka T, Sakamoto M, Nakagawa $H$, et al. Diffusion tensor tractography of the Meyer loop in cases of temporal lobe resection for temporal lobe epilepsy: correlation between postsurgical visual field defect and anterior limit of Meyer's loop on tractography. AJNR Am J Neuroradiol 2008;29:1329-34

27. Chen $\mathrm{X}$, Weigel $\mathrm{D}$, Ganslandt $\mathrm{O}$, et al. Prediction of visual field deficits by diffusion tensor imaging in temporal lobe epilepsy surgery. Neuroimage 2009;45:286-97

28. Wang YX, Zhu XL, Deng M, et al. The use of diffusion tensor tractography to measure the distance between the anterior tip of Meyer's loop and the temporal pole in a cohort from Southern China. J Neurosurg 2010;113:1144-51. Epub 2010 Aug 20

29. Hofer S, Karaus A, Frahm J. Reconstruction and dissection of the entire human visual pathway using diffusion tensor MRI. Front Neuroanat 2010;4:15

30. Clatworthy PL, Williams GB, Acosta-Cabronero J, et al. Probabilistic tractography of the optic radiations: an automated method and anatomical validation. Neuroimage 2010;49:2001-12

31. Sherbondy AJ, Dougherty RF, Napel S, et al. Identifying the human optic radiation using diffusion imaging and fiber tractography. J Vis 2008;8:1-11

32. Yogarajah M, Focke NK, Bonelli S, et al. Defining Meyer's loop-temporal lobe resections, visual field deficits and diffusion tensor tractography. Brain 2009; 132:1656-68

33. Tournier J-D, Calamante F, Connelly A. Robust determination of the fiber orientation distribution in diffusion MRI: non-negativity constrained superresolved spherical deconvolution. Neuroimage 2007;35:1459-72 